\title{
The Radiance Standard RASTA of DLR's calibration facility for airborne imaging spectrometers
}

\author{
T. Schwarzmaier, A. Baumgartner, P. Gege, C. Köhler, K. Lenhard \\ Deutsches Zentrum für Luft- und Raumfahrt (DLR), \\ Earth Observation Center, Remote Sensing Technology Institute, \\ Oberpfaffenhofen, 82234 Wessling, Germany
}

\begin{abstract}
The German Aerospace Center (DLR) operates the Calibation Home Base (CHB) as a facility for the calibration of airborne imaging spectrometers and for field spectrometers. Until recently, absolute radiometric calibration was based on an integrating sphere that is traceable to SI units through calibration at the German Metrology Institute PTB. However, the stability of the radiance output was not monitored regularly and reliably. This was the motivation to develop a new radiance standard (RASTA) which allows monitoring in the wavelength range from 380 to $2500 \mathrm{~nm}$. Radiance source is a diffuse reflector illuminated by a tungsten halogen lamp. Five radiometers mounted in a special geometry are used for monitoring. This setup improves twofold the uncertainty assessment compared to the previously used integrating sphere. Firstly, lamp irradiance and panel reflectance have been calibrated at PTB additionally to the radiance of the complete system. This calibration redundancy allows to detect systematic errors and to reduce calibration uncertainty. Secondly, the five radiometers form a redundant control system to measure changes of the spectral radiance. This enables long-time monitoring of the radiance source including assessment of the uncertainty caused by aging processes. Further advantages concern the reduction of periods of non-availability, applicability to sensors with larger field of view, and the possibility to alter intensity and spectral shape in a well-known way by exchanging the reflector. RASTA has been calibrated at PTB in November 2011 in the wavelength range from 350 to $2500 \mathrm{~nm}$
\end{abstract}

\section{MECHANICAL SETUP}

The mechanical configuration of RASTA is shown in Fig. 1. Light source is a 1000-W FEL tungsten-halogen lamp (Gamma Scientific Model 5000-16C), which is widely used as an irradiance standard. It illuminates perpendicularly a reflectance panel with the dimension $25 \mathrm{~cm} \times 25 \mathrm{~cm}$ (1). In order to minimize stray light contamination, the lamp is installed inside a lamp housing (2). The lamp housing is mounted moveably on a bar (3) to adjust the distance between lamp and reflectance panel. The distance can be measured accurately by mounting temporarily a lamp alignment jig instead of the lamp. A mounting adapter for measurement devices (4) is installed in front of the reflectance panel. It provides space for seven instruments arranged on a half circle around the optical axis, which is the line perpendicular to the panel center. This setup allows all instruments to look at the panel at the same spot from the same distance and in the same angle of $45^{\circ}$ to avoid differences in the radiance caused by spatial inhomogeneities. Five of the adapter spaces are used for radiometers $(5,6)$ which monitor the stability of the system. The two horizontal spaces left and right of the panel (7) can be used for 
additional sensors, e.g. spectrometers which shall be calibrated. For radiometric calibration of large sensors, these can be placed besides the system using separate mounts.

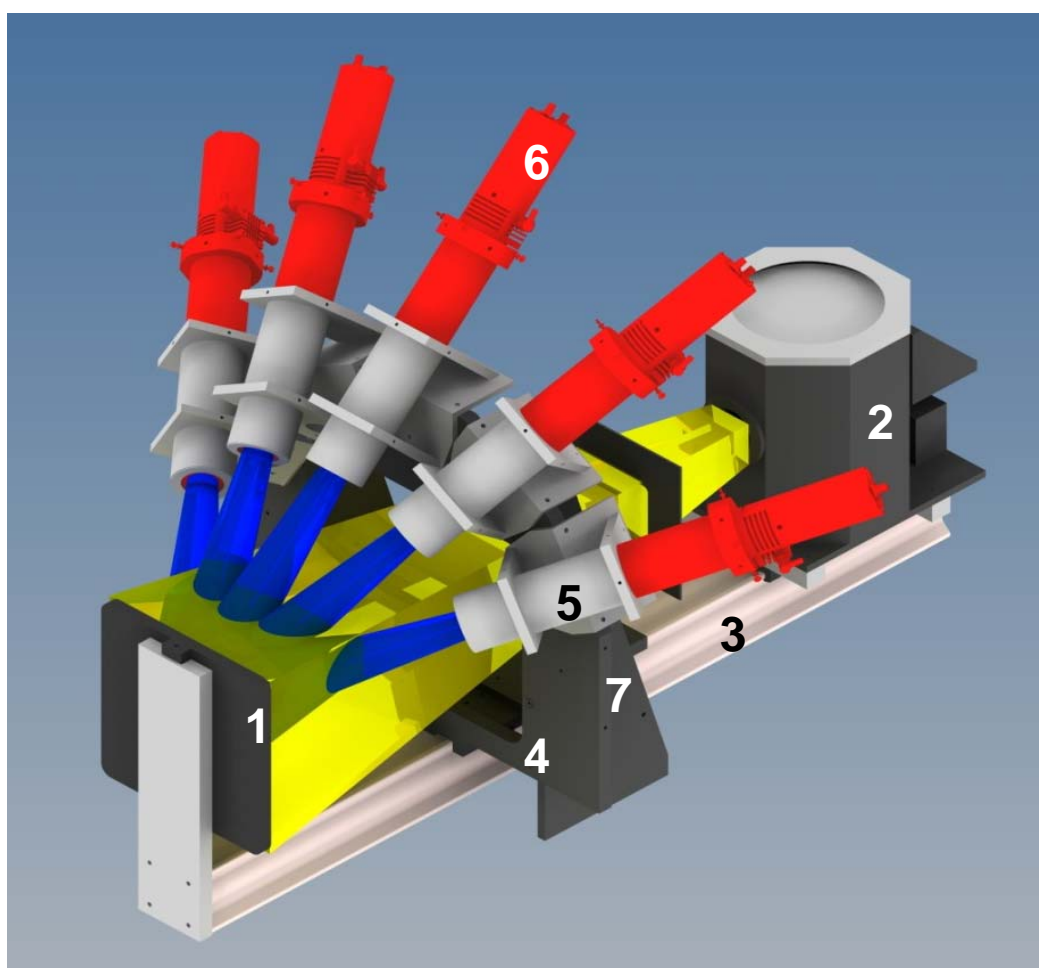

Fig 1: Mechanical set-up of RASTA. (1) Reflectance panel, (2) lamp housing, (3) bar, (4) mounting adapter for measuring devices, (5) mounting adapter for radiometer, (6) radiometer, (7) space to mount additional sensor. Yellow: beam illuminating reflectance panel, blue: field of view of radiometers

\section{ADVANTAGES OF RASTA}

\subsection{Monitoring and correction of radiance changes.}

The five radiometers form a redundant system to measure changes of the spectral radiance. Such changes are usually caused by aging of the lamp or contamination of the reflectance panel. Since the two causes have different wavelength dependency, they can be identified. If panel reflectivity has changed, the panel's reflectance spectrum has to be measured again. If lamp irradiance has changed, the actual radiance spectrum can be determined since changes of lamp irradiance are associated with changes of the lamp filament temperature. According to Planck's law, the irradiance changes (intensity and spectral shape) are determined by a single parameter (temperature), i.e. the five filter radiometers provide five-fold redundancy for measuring the irradiance changes.

\subsection{Redundant calibration}

Additionally to the radiance of the complete system, also lamp irradiance and panel reflectance have been calibrated at PTB. This redundancy allows to detect errors and reduces calibration uncertainty. 


\subsection{Size and shape.}

The reflectance panel Labsphere SRT-99-100 has an area of $25 \mathrm{~cm} \times 25 \mathrm{~cm}$, while the exit port of the integrating sphere is $20 \mathrm{~cm} \times 4 \mathrm{~cm}$. The increased size makes the new source more useful for sensors with large field of view, and the square shape allows to illuminate symmetrically the entrance aperture of sensors with circular field of view like field spectrometers and cameras.

\subsection{Flexible radiometry}

Radiance sources of different intensity and spectral shape can be realised easily by exchanging the reflectance panel or changing the distance between lamp and panel. Like this it is possible to adjust the radiance stepless and with constant spectral shape. Also doped or coloured panels can be used.

\subsection{Reduced complexity}

The radiance reflected from a panel that is illuminated by a single lamp can be calculated easily, while the radiance emitted from an integrating sphere is difficult to model since neither the coating nor the lamps can be characterised if the sphere is not disassembled. Integrating spheres are frequently used in combination with filters; however, these reflect some radiation back into the sphere, and the induced changes of sphere brightness are difficult to estimate. Modifications of RASTA can be modeled easier, e.g. exchange of lamp or panel.

\subsection{Maintenance}

Re-calibration of a radiance source is necessary when its spectral radiance differs significantly from that during calibration. The main cause for changes are the lamps. Since RASTA uses a single lamp while the previously used integrating sphere uses four, the probability of lamp failure is reduced and hence the interval of lamp exchange. Because a calibrated lamp is available as backup, it can be installed any time, eliminating periods of non-availability. Since the spectral radiance can be calculated using the irradiance of the lamp and the reflectance of the panel, only new components need to be calibrated, but not the entire system. The set-up of Fig. 1 allows further to calibrate the spectral irradiance of spare lamps by attaching the transfer spectrometer on the mounting adapter. 


\section{MONITORING SYSTEM}

Stability is monitored using five radiometers which cover the wavelength range from 350 to $2500 \mathrm{~nm}$, shown in Fig 2. These highly stable detectors (Gamma Scientific series TIA-3000) with a large dynamic range of 8 decades have been developed by Gamma Scientific in cooperation with NIST for the purpose to perform SI traceable radiometric calibration at high accuracy. Three of the radiometers use silicon detectors and optical filters to define the wavelength range (ranges: $320-500 \mathrm{~nm}, 450-650 \mathrm{~nm}, 650-1050 \mathrm{~nm}$ ), one an InGaAs detector (range: 850-1700 nm), and one an Extended Range InGaAs detector (range: 850-2500 $\mathrm{nm})$.

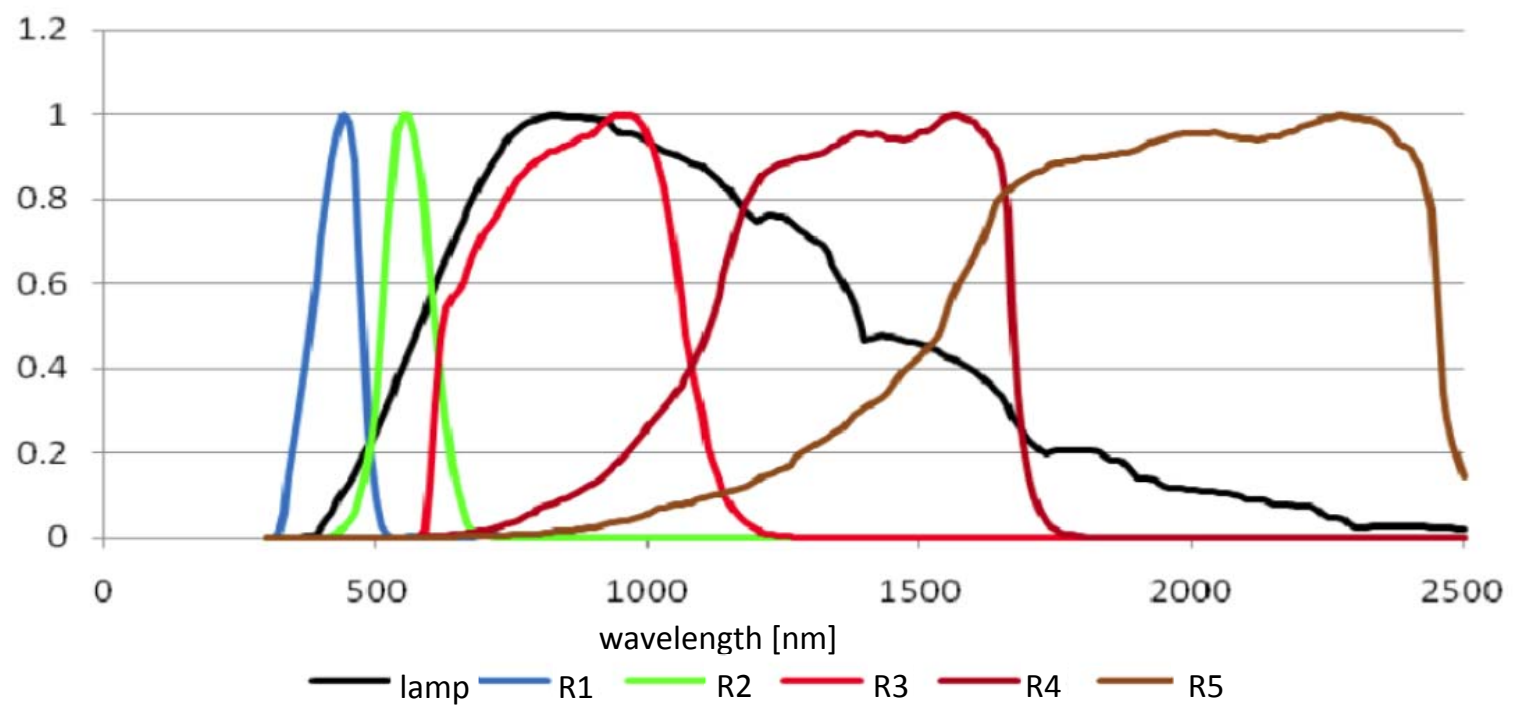

Fig 2: Response curves of the radiometers covering the lamp spectrum in the wavelength range of 350 to $2500 \mathrm{~nm}$

\section{CALIBRATION}

RASTA has been calibrated at PTB in November 2011 in the wavelength range from 350 to $2500 \mathrm{~nm}$ (Fig 3). The expanded uncertainty $(\mathrm{k}=2)$ is below $1.7 \%$ in the range $525-1700 \mathrm{~nm}$ and increases steadily towards shorter and longer wavelengths $(3.4 \% \text { at } 350 \mathrm{~nm}, 15 \% \text { at } 2500 \mathrm{~nm})^{4}$. The system is applied primarily for the radiometric calibration of spectrometers. One of these (SVC HR-1024) is used as transfer spectrometer to calibrate radiance sources operated in the CHB. By calibrating the transfer spectrometer shortly before usage and using the radiometers as independent detectors, the radiance spectrum of unknown sources can be measured at an uncertainty that is only slightly higher than that of RASTA. 


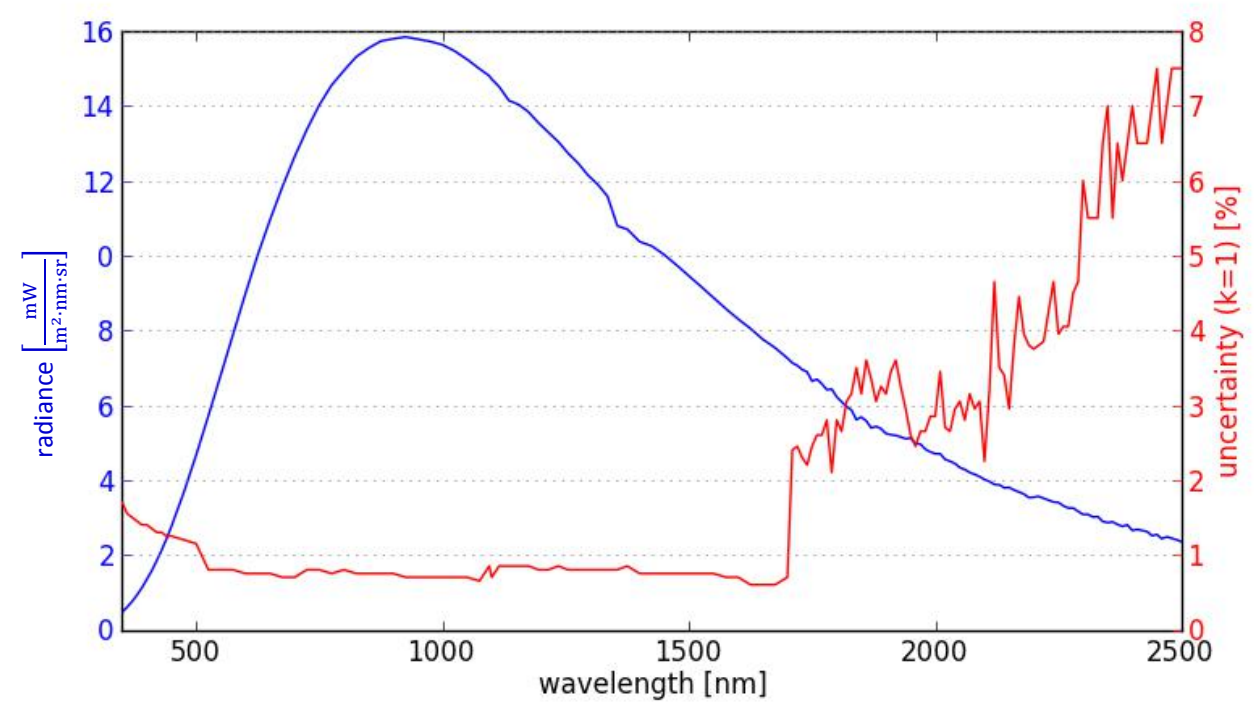

Fig 3: Spectral radiance of RASTA according to calibration at PTB in 2011

\section{CONCLUSION}

RASTA is the new reference radiance standard of the Calibration Home Base (CHB) of the German Aerospace Center. It was calibrated at the German Metrology Institute PTB and is thereby traceable to SI units. Because of redundant calibration and accurate monitoring it is a very reliable standard with a small uncertainty that can be used for calibration of radiance sources and sensors in the spectral range from 350 to $2500 \mathrm{~nm}$. To calibrate radiance sources, a transfer spectrometer is used which is calibrated with RASTA shortly before to minimize errors caused by drifting of the instrument. This enables CHB to provide a number of well and consistent calibrated radiance sources which are used to calibrate airborne sensors and field spectrometers of different size and viewing geometry. Future improvements will focus on the reduction of uncertainty of RASTA from 1800 to $2500 \mathrm{~nm}$, and on quantifying the uncertainties of the transfer spectrometer in measuring spectral radiance.

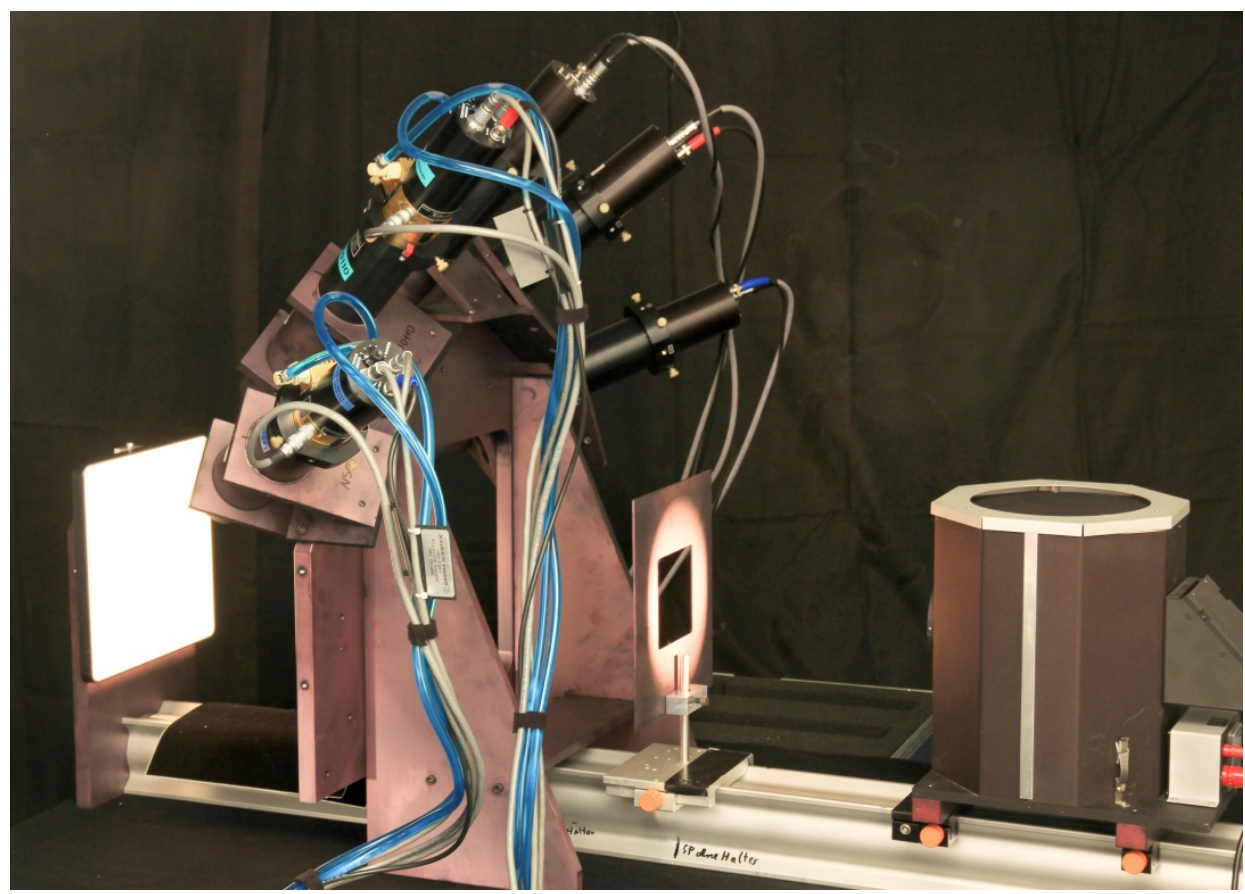

Fig 4: RASTA in use 


\section{REFERENCES}

1. Gege, P., Fries, J., Haschberger, P., Schötz, P., Schwarzer, H., Strobl, P., Suhr, B., Ulbrich, G. \& Vreeling, J. (2009). Calibration facility for airborne imaging spectrometers. ISPRS Journal of Photogrammetry \& Remote Sensing 64, 387-397.

2. Taubert, R. D., Monte, C., Gutschwager, B., Hartmann, J. \& Hollandt, J. (2009). Traceable Calibration of Radiation Sources from the Visible to the Far Infrared for Space Borne Applications at PTB. Proc. of SPIE 7474, 747413-1-747413-12.

3. Gege, P., Fries, J., Haschberger, P., Lenhard, K., Schötz, P., Ch. J. Schwarz, Ch. J., Schwarzmaier, T. (2010): Concept for improved radiometric calibration of radiance sources at the CHB facility. Proc. Hyperspectral Workshop, March 17-19, 2010, ESRIN, Frascati, Italy.

4. Taubert, D.R., Hollandt, J., Sperfeld, P., Höpe, A., Pape,S., Gege,P., Schwarzmaier, T., Lenhard, K., Baumgartner, A. (2012): Providing radiometric traceability for the Calibration Home Base of DLR by PTB. International Radiation Symposium, August 06-10, 2012, Berlin, Germany

5. Baumgartner, A., Köhler, C., Lenhard, K., Gege, P., Schwarzmaier, T. (2012): Characterisation methods for the hyperspectral sensor HySpex at DLR's calibration home base. SPIE Remote Sensing Europe, September 24-27, 2012, Edinburgh, United Kingdom 